\title{
Discourse and Conversation
}

Ribut Wahyudi, UIN M2I Malang,

March 28, 2013.

Email: ribut@bsi.uin-malang.ac.id 


\section{Conversation Analysis (CA)}

- $\mathrm{CA}$ is one of the major studies of discourse CA is an approach to the analysis of spoken discourse with regard to how people manage daily conversational interaction.

Some aspects to examine: adjacency pairs, preference organization, turn taking, feedback, repair, conversational opening and closings, discourse markers and response tokens.

CA works with recordings of spoken data and carriesout careful and fine-grained analyses. 


\section{Background to CA}

- Began in the early 1960 s at the University of California Leading scholars Sacks, Schegloff \& Jefferson It's from Sociology

- It takes less "linguistic view" compared to some other forms of DA

CA is interested in how "social worlds" are jointly constructed and recognized by speakers as they engage in conversation. The focus of early work is casual conversation.

- 슬 Current foci: doctor-patient interaction, legal hearings, news interviews, psychiatric interviews, and interactions in courtroom and classrooms. 


\section{Issues in CA}

- Ordinary conversation = basic form of talk Conversation = the main way for people to come together, exchange information, \& maintain social relation.

A Key feature of CA is the primacy of data as the source of information

Analyses in CA do not incorporate speaker's reflections on their interaction, field notes or interviews

CA focuses on the analysis of the text for argumentation and explanation.

- CA avoids assumptions about analytical categories in the analysis of conversational data. 


\section{Conts...}

- Conversation Analyst look for phenomena which regularly occur in the data and then make the point for further investigation.

- The interest of CA is primarily fine-tuned analysis of the sequence, structure and coherence of conversations.

Conversation Analysis aims to demonstrate how participants both produce and respond to evolving social contexts, using conversational, rather than contextual data as the source for the claims. 


\section{Transcribing and coding conversation analysis data}

- In CA, the transcription of data is also analysis Texts are recorded, then analyzed as they are recorded Knowing the particular features such as the use of increased pitch, or particular sequence of utterances becomes the starting point of the analysis. The analyst listens and transcribes to see how frequently this aspect of the conversation occurs and importantly, if speakers respond to it in the same way each time it occurs. In this way, the analysis aims to understand how speakers manage their conversational interactions. 


\section{Transcription conventions (based on Jefferson (2004)}

NOW loud sounds relative to the surrounding talk prolongation of the immediately prior sound a brief interval (about a tenth of a second) within or between utterances

(0.5) the time elapses (by tents of seconds) between the end of the utterance or sound and the start of the next utterance or sound

now stress

rising intonation

Falling intonation

unfinished intonational contour (see. p.109) 


\section{Sequence and structure in conversation}

- Opening conversations e.g. in telp conversation, radio call program see p.111

Closing conversations

Turn taking

Adjacency pairs = utterances by two successive speakers in a way that the second utterance is identified as related to the first one as an expected follow-up to that utterance. However, there is a case in which the first speaker is challenged by the second speaker. See. p. 115. 


\section{Adjacency pairs across cultures}

- Beal (1992): Did you have a good weekend \# adjacency pairs and the stage of conversation

"Hello" can either be a summons in a telephone call or a response to a summons, or it means greeting in the street.

"Thanks" is a response of compliment or a response to an offer of service.

An utterance thus play more than one role in a conversation. 


\section{Preference Organization}

- See common adjacency pairs and typical preferred and dis-preferred second pair parts (Levinson, 1983) see. P.117.

- Insertion sequence

\# Feedback = speaker's response to what is being said e.g. : 'response token' such as: 'mmm', 'yeah' etc These response may function different thing (an acknowledging function, to prompt a topic change, a recycling of topic, or disprefered action. 


\section{Repair}

- Self repair \& Other repair

\# Gender and Conversation Analysis

Weatherall (2002) "gender noticing"

Stokoe (2003) "membership categorization analysis" (see.page.120).

\# CA and L2 conversation

Markee (2000) CA can be used as a tool for analyzing and understanding the acquisition of second language. 


\section{Criticism of CA}

- 'monolithic' (Baxter, 2002)

Hammersley (2003) CA is problematic as it views itself as self-sufficient research tool.

"analyst" is spectator in the conversation not participant, it is thus, not really possible for us to know how the participants view the conversation unless we ask them etc.

A sample study: refusals $=>$ accept to the point, while refusing sth: needs strategy as it might be FTA. 


\section{References}

- Paltridge, B. 2006. Discourse Analysis. London: Continuum. Other related materials. 


\section{Thank You}

\section{Kingsoft Office}

Make Presentation much mose fun

(5) @Kingsoft_Office

ff kingsoftstore 